\title{
CMAF - CHRIS Morphological Adaptive Filter
}

\author{
Przemysław Kupidura
}

Faculty of Geodesy and Cartography, Warsaw University of Technology

Pl. Politechniki 1, 00-661 Warsaw, Poland

e-mail: p.kupidura@gik.pw.edu.pl

\begin{abstract}
The paper presents a new method of CHRIS images filtering. The presented algorithm is based on mathematical morphology operations and allows to correct the main CHRIS images noise types, like missing pixels and vertical stripes caused by a malfunctioning of the device. the algorithm is preceded by the brief discussion on the nature of the noise and the basis of mathematical morphology. The resulting images are compared to the results of application of other types of CHRIS-dedicated algorithms (Settle methods).
\end{abstract}

Key words: CHRIS/PROBA, image filtration, mathematical morphology

\section{Introduction}

This paper presents the author-made adaptive algorithm created to filter specific noise which occasionally occurs on CHRIS images. CHRIS (Compact High Resolution Imaging Spectrometer) is a multi-angle hyperspectral spectrometer placed on the PROBA (Project for On-Board Autonomy) satellite platform launched on 22 October 2001. CHRIS/PROBA mission, funded by European Space Agency (ESA) and British National Space Center (BNSC), which thanks to its flexibility (five different spatial and spectral resolution modes) and availability, became an important source of data for land and environmental applications (Osińska-Skotak et al. 2004). However, occasionally, image errors, caused by a device imperfection, may occur. There are two main types of the mentioned errors: horizontal, one-pixel thin "bad lines", comprising correct and bad pixels, intertwined, and vertical, bright or dark stripes of varied thickness (from one to several pixels) (Kupidura 2010 et al.). There is a popular filter, developed by Settle (Cutter 2004; Garcia \& Moreno 2004), allowing correction of some kinds of horizontal "bad lines", and also, to suppress the vertical noise. Usually the Settle method works with a very high efficiency, though the most important advantage of this filter is that it doesn't cause any blur or detail suppression. However, sometimes the structure of the objects on an image, like "vertically" oriented (dark) rivers, or (bright) clouds may be mistreated as the spoken errors, leading to an image being corrupted instead of being corrected. There are also other algorithms dedicated to CHRIS satellite images.

The proposed algorithm - CMAF (Chris Morphological Adaptive Filter) is a mathematical morphology based algorithm which allows the elimination of all types of errors occurring on CHRIS images. It consists of 3 consecutive steps, dedicated to different error types: "black bad lines", "bright bad lines" and erroneous vertical stripes. It is based on several, relatively simply morphological operations, which may be applied using free software (in this case BlueNote), an open-source software, developed basing on the author design, dedicated to morphological operations). The filter has an adaptive character, which means, that firstly, the occurrence of the noise is tested, and the result of the filtering operations is only applied on erroneous lines (horizontal or vertical), which eliminates or at least minimizes the filter influence on the correct parts of the image. 
The result of the CMAF algorithm shows highly efficient noise detection and suppression. Thanks to its adaptive character, it preserves edges of objects in an image. The visual analysis of the results, in comparison to other filter types shows that CMAF is slightly less effective than the Settle method, but on the other hand it doesn't cause the errors, typical for the Settle method. What is also important is that CMAF completely eliminates all types of "bad lines" errors, while there is no other algorithm dedicated to "bright bad lines".

This paper shows, that CMAF might be an interesting alternative to other, popular algorithms dedicated to the filtering of CHRIS images.

\section{Theory}

In this section the fundamental functions of mathematical morphology used in the algorithm presented in this paper are briefly described.

Mathematical morphology is a set theory approach, developed by J. Serra and G. Mathheron (Matheron 1967, 1978). It provides an approach to processing digital images based on geometrical shape.

Two fundamental morphological operations - erosion and dilation are based on Minkowski operations. There are two different types of notations for these operations: Serra/ Matheron notation (Serra 1982, 1986, 1988) and Haralick/ Sternberg notation (Sternberg 1986; Haralick 1987). They are, however, defined for binary images, and do not fit grey-scale image operations. Therefore we propose (after Nieniewski 1998) a definition working for both: binary as well as grey-scale images. Erosion may be defined as follow:

$$
\varepsilon_{B}(f)=\inf \{g(f-y), y \in B\}
$$

where: $\varepsilon_{B}(f) \varepsilon_{B}(f)$ is an erosion of a function (image) $f$ using a structuring element (SE) $B B$, so the result of an erosion is the smallest value amongst all images (functions) created by all offsets by the elements opposite to the elements of the SE.

Analogically, dilation may be defined as follows:

$$
\delta_{B}(f)=\sup \{g(f+y), y \in B\}
$$

where: $\delta_{B}(f) \delta_{B}(f)$ is a dilation of a function (image) $f$ using a structuring element $B B$, so the dilation is the highest value of all created by all offsets by the elements of the SE.

Two other principal operations, opening and closing, are simple sequences of erosion and dilation. Opening is defined by the following equation:

$$
\gamma_{\mathbf{B}}(f)=\delta_{\mathbf{B}}\left(\varepsilon_{\mathbf{B}}(f)\right) \gamma_{\mathbf{B}}(f)=\delta_{\mathbf{B}}\left(\varepsilon_{\mathbf{B}}(f)\right)
$$

and closing as:

$$
\varphi_{\mathrm{B}}(f)=\varepsilon_{\mathrm{B}}\left(\delta_{\mathrm{B}}(f)\right) \varphi_{\mathrm{B}}(f)=\varepsilon_{\mathrm{B}}\left(\delta_{\mathrm{B}}(f)\right)
$$

where $\gamma_{\mathrm{B}}(f) \gamma_{\mathrm{B}}(f)$ and $\varphi_{\mathrm{B}}(f) \varphi_{\mathrm{B}}(f)$ are opening and closing of a function (image) $f$ using a structuring element $B B$.

Opening and closing operations are very often used sequentially as so called alternate filters, combining their abilities to eliminate small dark (opening) and bright (closing) objects. Usually they are denoted as CO (closing-opening) or OC (opening-closing) filters:

$$
\begin{aligned}
& \mathrm{CO}_{\mathrm{B}}(f)=\gamma_{\mathrm{B}}\left(\varphi_{\mathrm{B}}(f)\right) \\
& \mathrm{OC}_{\mathrm{B}}(f)=\varphi_{\mathrm{B}}\left(\gamma_{\mathrm{B}}(f)\right)
\end{aligned}
$$

where $\mathrm{CO}(f) \mathrm{CO}(f)$ and $\mathrm{OC}(f) \mathrm{OC}(f)$ are, respectively, closing-opening and opening-closing filtration of function (image) $f$ using a structuring element $B$.

Opening and closing operations are presented in figure 1.

Another operation important for the research presented in this paper is a top-hat transformation. Briefly, its purpose is to eliminate big objects from the image, by simple subtraction the image after opening operations from the original image:

$$
\mathrm{TH}_{\mathrm{B}}(f)=f-\gamma_{\mathrm{B}}(f)
$$

where $\mathrm{TH}_{\mathrm{B}}(f) \mathrm{TH}_{\mathrm{B}}(f)$ is a top hat transformation of a function (image) $f$ using a structuring element $B$. Top hat transformation leaves only small objects (smaller than a SE) in the image, brighter (of higher value) than their neighborhood. There is also a dual operation, using a closing operation instead of opening. In this version we subtract the original image from the result of closing. To distinguish between these two operations we suggest calling one of them (presented in the equation above) simply: top hat, and the second one (based on closing) - bottom hat (which is a kind of a play of words). Please, bear in mind, that often they are also called, respectively: white top hat and black top hat. Bottom hat transformation may be presented as follow:

$$
\mathrm{BH}(f)=\varphi_{\mathrm{B}}(f)-f
$$

where $\mathrm{BH}_{\mathrm{B}}(f) \mathrm{BH}_{\mathrm{B}}(f)$ is a bottom hat transformation of a function (image) $f$ using a structuring element $B$. Bottom hat finds objects darker (of lower value) than the neighborhood, reversely to a top hat transformation. 

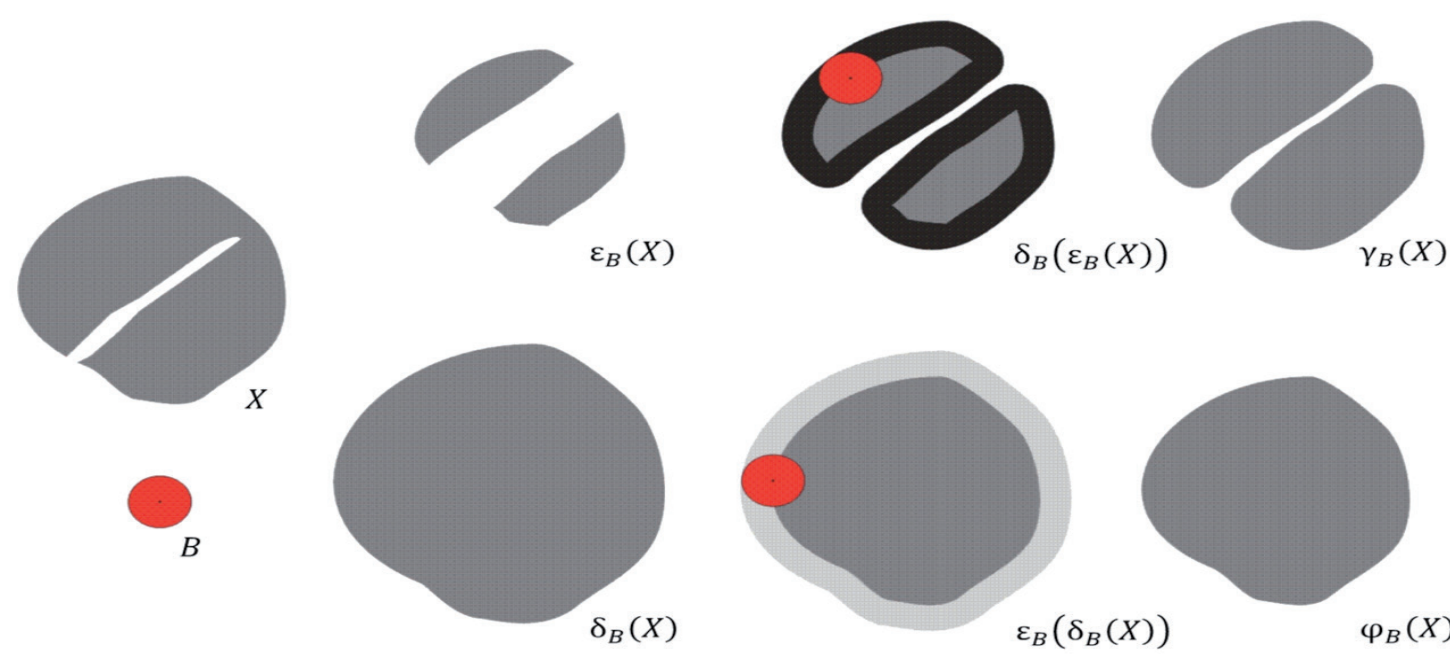

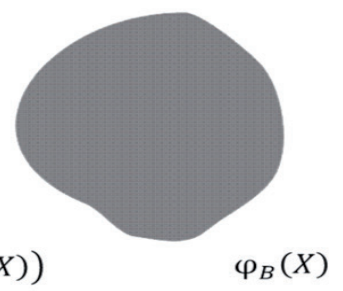

Figure 1. Erosion, dilation, opening and closing, using the structuring element B

The last kind of operation to be presented in this section is an operation with multiple SE (Stevenson \& Arce 1987; Song \& Delp 1990; Cheng \& Venetsanopoulos 1992; Vincent 1992, 1993). Actually it is not a separate operation, but rather a modification, which may be used for most morphological operations. It is based on decomposition of the SE into smaller elements and follows one of the equations presented below (in the example, respectively, opening and closing):

$$
\begin{aligned}
& \gamma_{G}(X)=\max _{\mathrm{B}^{\mathrm{k}} \in \mathrm{G}}\left(\gamma_{B^{k}}(X)\right) \\
& \varphi_{G}(X)=\min _{\mathrm{B}^{\mathrm{k}} \in \mathrm{G}}\left(\varphi_{B^{k}}(X)\right)
\end{aligned}
$$

where $\gamma_{G}(X) \gamma_{G}(X)$ and $\varphi_{G}(X) \varphi_{G}(X)$ are, respectively, opening and closing of an image $X$ using a multiple structuring element $G$, consisting of singular structuring elements $B^{k} B^{k}$.

This kind of operations allows the elimination of (or discovery if we use it for top/bottom hat transformations) objects taking into account possible specific structures (e.g linear). Figure 2 presents this concept.

As we can see, simple opening removes all objects smaller/thinner than the SE, while opening with multiple SE preserves linear structures. Depending on a multiple SE, other shapes and characteristics might be taken into account (Stevenson \& Arce 1987; Song \& Delp 1990; Cheng \& Venetsanopoulos 1992; Vincent 1992, 1993; Kupidura 2006; Kupidura et al., 2010)

Only a brief introduction to mathematical morphology is presented in this paper. Readers wanting to deepen
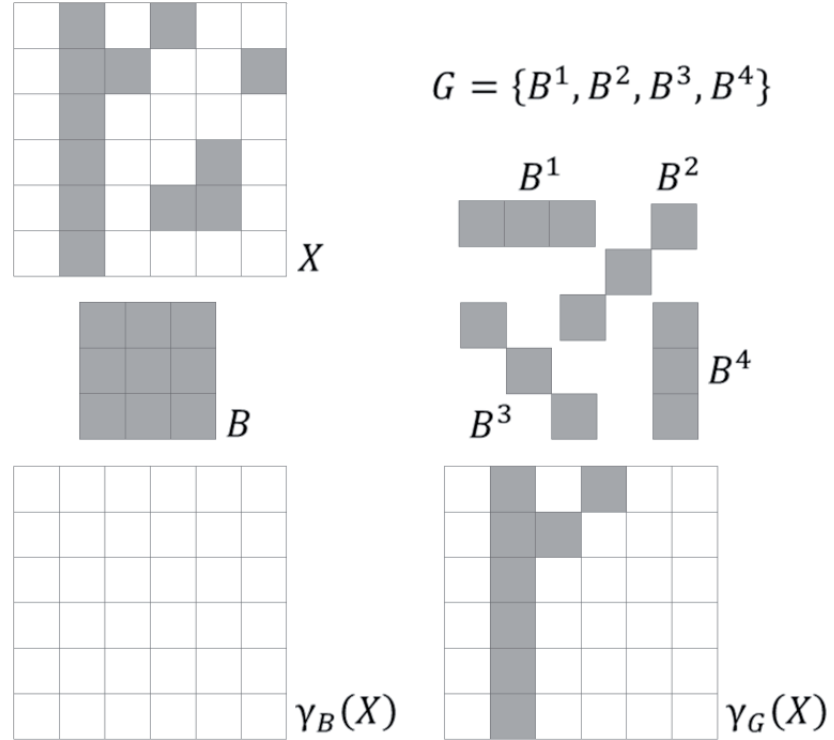

Figure 2. Opening with multiple SE, compared to simple opening

their knowledge of mathematical morphology are referred to the books and articles of mathematical morphology for an extended background to morphological operators (Serra 1982; Nieniewski 1998, 2005; Kupidura 2006; Kupidura et al. 2010). 


\section{Problem}

The CHRIS/PROBA mission is an important source of data for land and environmental applications. However, some CHRIS images are affected by noise and errors, caused by device imperfection.

There are two main types of the mentioned problems: horizontal, one-pixel thin "bad lines", comprising correct and bad pixels, intertwined, and vertical, bright or dark stripes of varied thickness (from one to several pixels) (Barducci et al. 2005; Gomez-Chova et al. 2008; Kupidura 2010). Horizontal bad lines, which we may treat as errors, as they are measurements "faults", might also appear in one of two versions: black (where bad pixels have value 0), and bright (where bad pixels have incorrectly high values). Let's present this problem in Figure 3.

The first kind of noise - black bad lines presented in Figure 4a seems relatively easy to eliminate, as bad pixels have the same (and unique) value -0 . The second kind, bright bad lines, are much more difficult to filter as the values of bad pixels vary - they are generally higher than they should be, but it is only possible to mark them out by comparing them to their neighbors.
Different kind of the CHRIS images imperfections appears as vertical stripes of different width and different brightness, and should be treated as noise, rather than an error, as it is caused by "normal" though "imperfect" device functioning The example of such noise is presented in Figure 4.

Here, also, the value of the stripes differ, so it is difficult to mark them out. The different width of the stripes additionally hampers this task.

As mentioned in the introduction, the Settle method allows the elimination of at least some of the problems listed above. Firstly, it removes black bad lines, secondly, it deals with vertical stripes, by analyzing all the vertical pixel columns, calculating their average values, and filtering the "chart" created this way (much more details in (Cutter 2004; Garcia \& Moreno 2004)) which allows the elimination of errors appearing in such a chart as the average values differing significantly from the values of the neighboring lines. In most cases, this method works very well, as we can see in Figure 5 (though, still we can see a bright bad line left in the filtered image).

Unfortunately, the Settle method might not work properly in some cases, when large, very bright (like clouds)

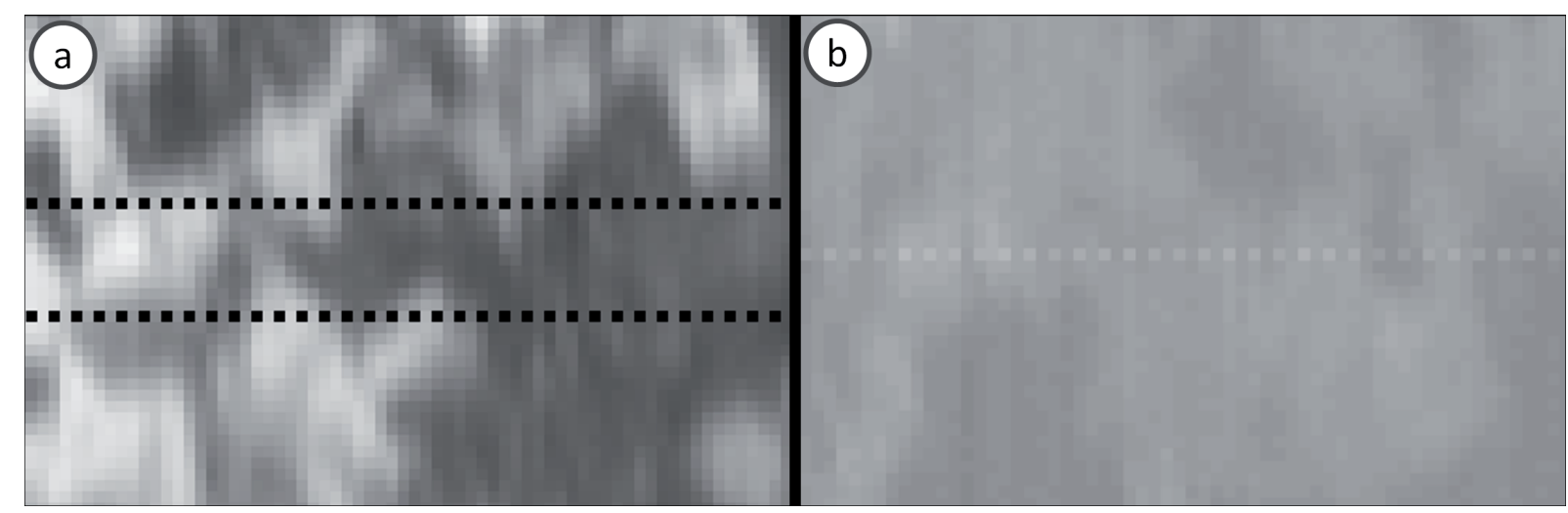

Figure 3. Bad lines, a) black b) bright

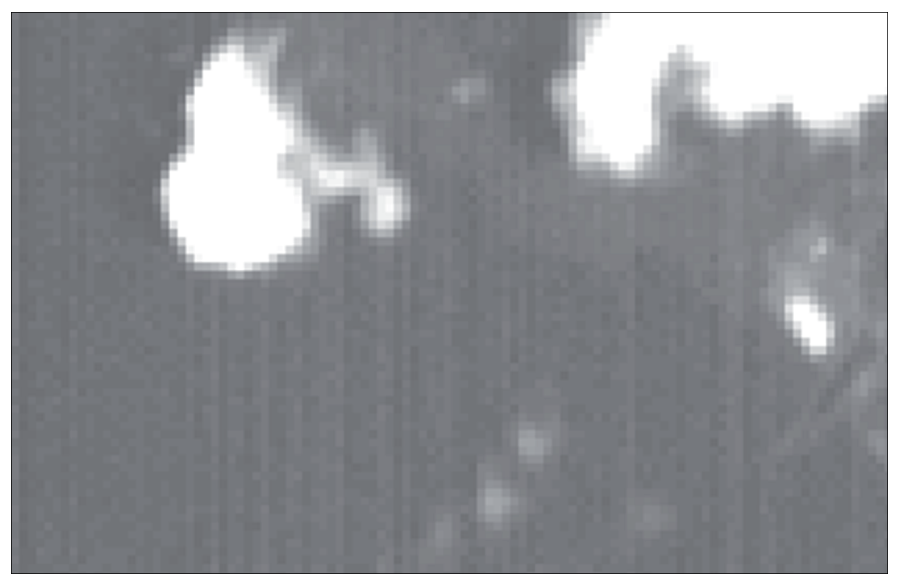

Figure 4. Vertical stripes in a CHRIS image 

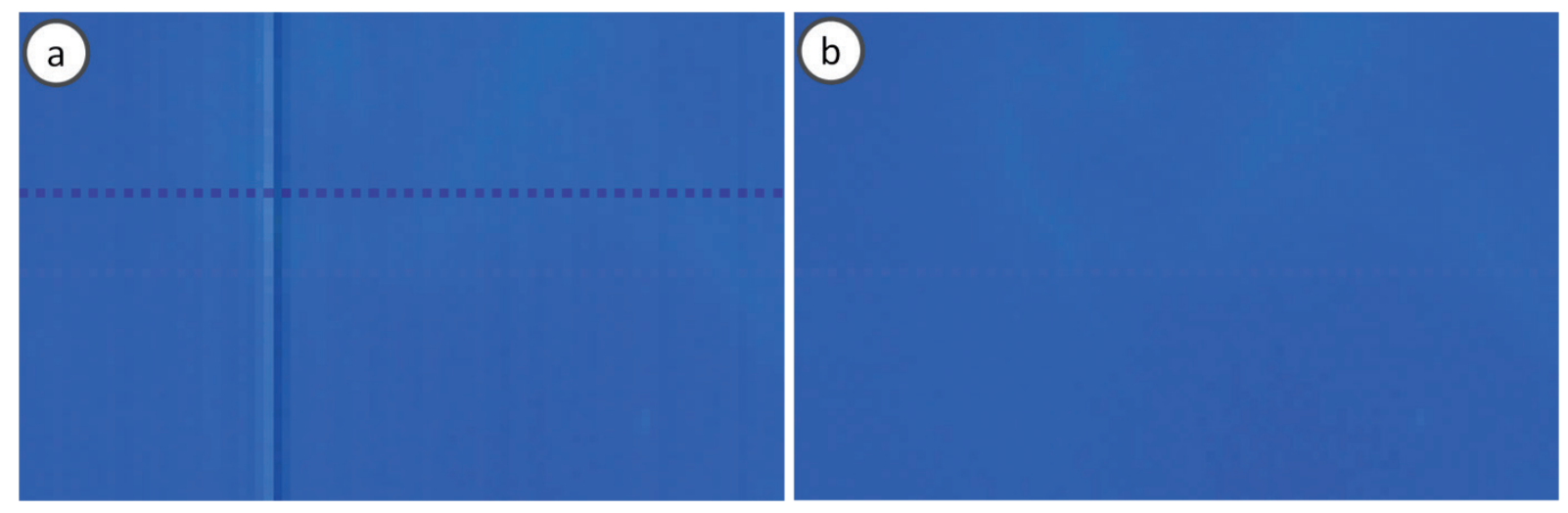

Figure 5. Result of Settle method application, a) original image, b) image after filtration using Settle method

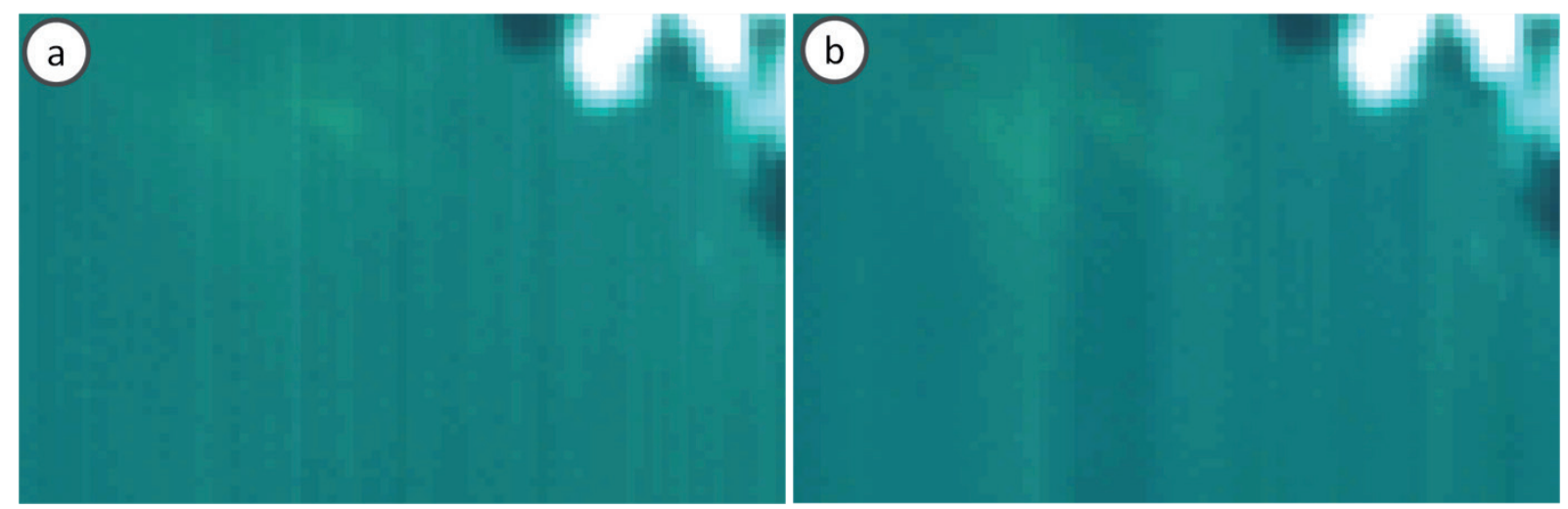

Figure 6. Result of Settle method application, a) original image, 6b) image after filtration using the Settle method

or very dark (like rivers) objects appear, and are oriented vertically (in the image). In such a case, average values of pixel columns are significantly increased or lowered, and the filtration algorithm might bring them closer to the average column value and in this way corrupt them. An example of such a situation is presented in Figure 6.

\section{Methods}

The filter being presented in this paper consists of three steps dedicated to three different types of noise, specific to CHRIS images. The first two steps are dedicated to bad lines (black and bright) and the third one is dedicated to the suppression of vertical stripes (which is the most difficult objective of the three). All three algorithms together form CMAF - CHRIS Morphological Adaptive Filter. The filter is morphological, as it is based mainly on mathematical morphology operations, and adaptive, because it only performs on the noise itself, previously marked out from an image, which limits the unwanted modification in the rest of the image.

Also, it applies different solutions depending on the "imperfection" type: horizontal "errors", as the result of the "total" (but temporary or local) malfunctioning of the device are replaced by the new pixel values, while vertical "noise" is corrected, rather than replaced, according to the value of the noise detected using the algorithm.

\subsection{Bad lines elimination}

Theoretically, bad lines (black as well as bright) could be easily removed using a median filter of a singular size (e.g. square $3 p \times 3 p$ ). One-pixels noise will be eliminated and will not affect neighboring pixels. However, this way, the whole image will be modified. To avoid such a situation, an adaptive approach is necessary. The approach presented below consists in firstly singling out the error pixels, and secondly, in applying modifications (filtration) only in the pixels singled out as noise.

\section{Black bad lines}

Black bad lines are relatively easy to correct. All erroneous pixels in them have value 0 , the value which practically does not occur in the other (correct) pixels in the image. The easiest way to remove this kind of noise is to distinguish all 0 value pixels in the image, and replace their 
values with the average value of two neighbor pixels: one above and one below (this approach is applied in the Settle method). However, below we propose the algorithm allowing the correction of bad lines, without changing possible singular correctly 0 -valued pixels (which is theoretically possible, especially after a histogram stretching).

First, we have to distinguish bad 0-valued pixels from the good ones. A bad lines characteristic is that they comprise correct and bad pixels, intertwined. Therefore, application of erosion using a flat, horizontal SE of $3 p \times 1 p$ size, creates a one-pixel-thin, whole-image-long horizontal line combined of 0 -valued pixels (Fig. 7b). Creation of such a line in any different case is unlikely, or, at least, very rare, as it requires dense properly 0 -valued pixels, without a gap between 0 -valued pixels bigger than one pixel, so we may assume, that the 0 -valued image-width-long lines indicate black bad lines. To eliminate shorter black lines, we can apply a dilation of very long, horizontal SE; real black bad lines will be preserved, as they are only combined of black pixels (Fig. 7c). Thanks to these operations we may assume, that pixels of value 0 in both, original and processed images are noise. Now, when we know exactly where they are (Fig 7d), we may change their values based on neighboring pixel values. The easy way to do this is to process the original image using a mean filtration with a vertical $1 \mathrm{p} \times 3 \mathrm{p}$ filtering window (Fig. 7e), and apply the obtained values only in bad pixels singled out before (Fig. 7f). This way, correct pixel values will not be changed.

Reassuming, we may present the algorithm of black bad lines correction as follows:

1. Erosion with a short horizontal SE $-3 p \times 1 p$.

2. Dilation with a long horizontal SE - 1531p x 1p in the case presented in this paper, but the length of the element depends on the size of the image (should not be shorter than the width of an image being processed).

3. Sum of two images: the one obtained during step 2 and the original one.

4. Mean filtration of the original image with a short vertical filtering window $-1 p \times 3 p$.

5. Application of the result of a mean filtration (step 4) in the pixels with 0 value as a result of step 3 .

The algorithm presented above is illustrated in figure 7 .

\section{Bright Bad Lines}

Bright bad lines are more difficult to correct, as their distinction from correct lines is more complex. It is because there is no specific value that might indicate a bad pixel of this kind. As seen in figure 3b, they are, however, significantly brighter than the neighboring pixels, and this is the characteristic we must use.

First, we have to indicate the pixels brighter than their neighbours. We do this by using a top hat transformation with multiple SE (Fig. 8b). Differently than in a case of black bad lines, we do not single out all noise pixels, as good pixels in neighboring horizontal lines (but also from the same line) might have higher values and also, we may distinguish a significant number of good pixels. To filter them out and, simultaneously, and to connect genuinely bad pixels, to make their correction in the following steps easier, we may use alternate $\mathrm{CO}$ filtering using a short horizontal SE (Fig. 8c) assuming, that most of the bad pixels have been singled out in the previous step, thanks to the filtration, knowing that they will create long non-zero lines. A significant amount of other pixels indicated by top hat transformation will be eliminated, while the remaining will create relatively short lines, which may be deleted using an erosion with a horizontal one-pixel-thin SE. The SE cannot be too long, as it may also delete the real bad lines we want to show. In the test being presented in this paper, an element of a size $99 \mathrm{p} \times 1 \mathrm{p}$. After this process we may assume that non-zero pixels indicate the presence of the bad lines. Dilation with a very long horizontal SE allows us to extend these lines for the whole width of the image (Fig. 8d). The next step is to form an intersection of the image obtained in the last process and the image after top hat transformation. As a result we obtain an image, where all the non-zero pixels indicate bad pixels in bright bad lines (Fig. 8e). Now it is easy to correct the image, replacing the wrong values with values from the image processed using a mean filtration mentioned in the previous section (Fig. 8f). We can assume the above using the following list:

1. Top hat transformation with multiple SE (size $3 p \times 3 p$ ).

2. Alternate CO filtration using an SE of size $3 p \times 1 p$.

3. Erosion using a long horizontal SE (99p x 1p)

4. Dilation using a very long horizontal SE - 1531p x $1 \mathrm{p}$ in this case, but the length of the element depends on the size of the image (it should not be shorter than the width of the image being processed).

5. Intersection of the images obtained in steps 1 and 4 .

6. Mean filtration of the original image with a short vertical filtering window $-1 \mathrm{p} \times 3 \mathrm{p}$.

7. Application of the result of the mean filtration (step 4) in the pixels with 0 value in the result of step 3 .

The results of the algorithm presented above is shown in Figure 8.

\subsection{Vertical Stripes}

This kind of noise consists of vertical distortion, i.e. oriented along the satellite movement vector. Due to the character of this noise (stripes causing increasing or decreasing pixel values) it would appear that an alternate $\mathrm{CO}$ or OC filtration might be successful. However, it is not recommended to use this filtration without proper preparation - the filtration should be preceded by the determination of noise, in order to limit the modification caused by the filtering. 

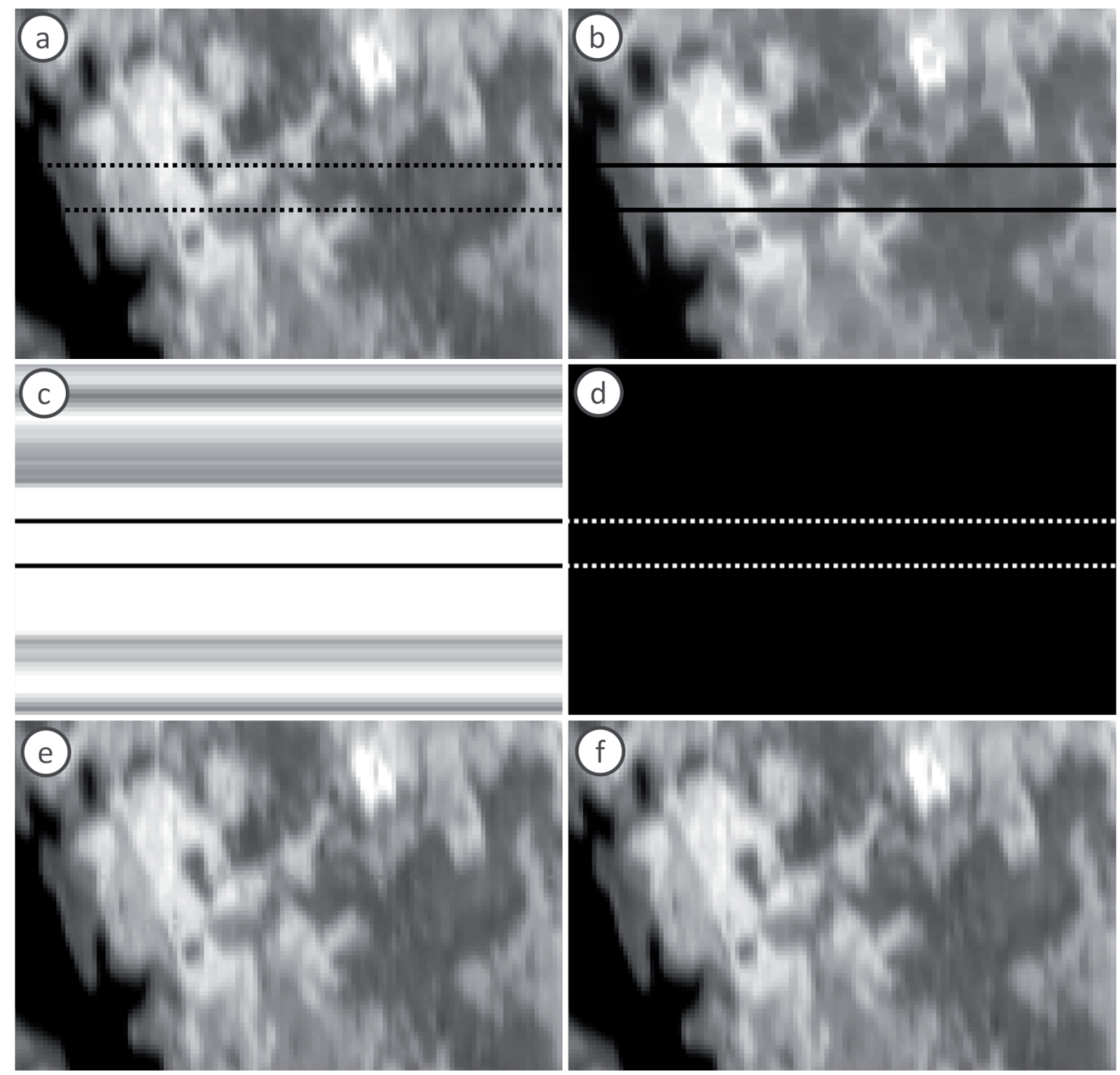

Figure 7. Consecutive steps of black bad lines elimination algorithm, a) original image with bad lines, b) erosion (step 1), c) dilation (step 2), d) missing pixels (step 3), e) mean filtration of the original image (step 4), f) final result of the process (step 5)

First, should break down the noise we speak about into components: bright stripes and dark stripes. Each of them will be corrected separately. First, we will correct the bright noise component, and then the dark one, but the order we propose is not obligatory - the reverse order has the same effect (the result we obtain may differ, but this an inconsequential matter).

Regarding the noise characteristic, the top hat transformation using a small horizontal SE (e.g. 3p x 1p) should reveal most of the affected stripes. There will also be other pixels indicated as non-zero-valued as a result of this pro- cess (Fig. 9b), so it is important to eliminate them. This can be achieved by erosion with a relatively long vertical SE (here $-1 \mathrm{p} \times 13 \mathrm{p}$ ), because the genuinely affected stripes form longer lines than pixels singled out accidentally, so this process eliminates all non genuine noise from the image (Fig. 9c). Assuming that non-zero pixels have been recognized, we may now lengthen them using a dilation with a very long vertical SE (this should not be shorter than the number of pixels in a column). After binarization of this image, we obtain the bright noise stripes. The result is presented in figure 9d. Now, when the 'noised' stripes 


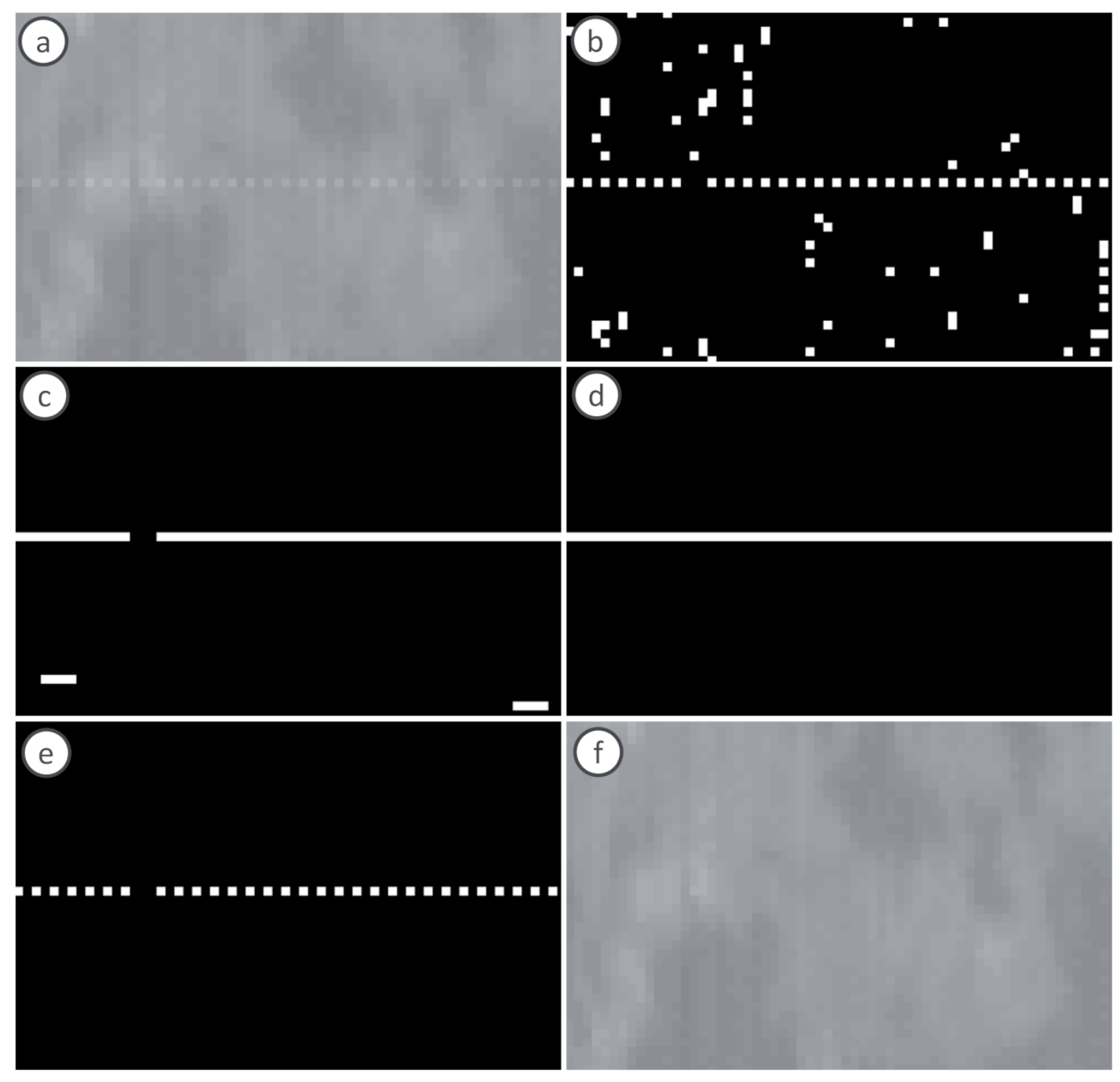

Figure 8. Consecutive steps of bright bad lines elimination algorithm a) original image, b) top-hat transformation with multiple SE (step 1), c) alternate CO filtration (step 2), d) dilation (step 4), e) bad pixels (step 5), f) final result (step 7)

(or at least, a significant part of them) are marked, we can apply a modification in order to correct them. Multiplying two images: one with bright stripes marked and one after top hat transformation, will result in the image showing the values we may assume as 'noised' (Fig. 9e), so subtracting them from the original image will suppress the (bright) noise from it (Fig. 9f).

The part of the algorithm dedicated to elimination of dark stripes is very similar bearing in mind that the top transformation is replaced by bottom hat transformation, and the detected noise is added to the image after the first part of the algorithm, not subtracted from the original..The final result of the algorithm with relation to 'noised' dark stripes and the image after their suppression is presented in Figure $9 \mathrm{~g}$ and $9 \mathrm{~h}$.

We may assume these following steps:

1. Top-hat transformation using a short horizontal SE (3p x 1p).

2. Erosion with a vertical SE (1p x 13p).

3. Dilation using a long vertical SE (1p x 1495p - depending on the number of lines in the image.

4. Binarization of the image with a threshold of 1 .

5. Product of the result of binarization and top-hat transformation (step 1). 


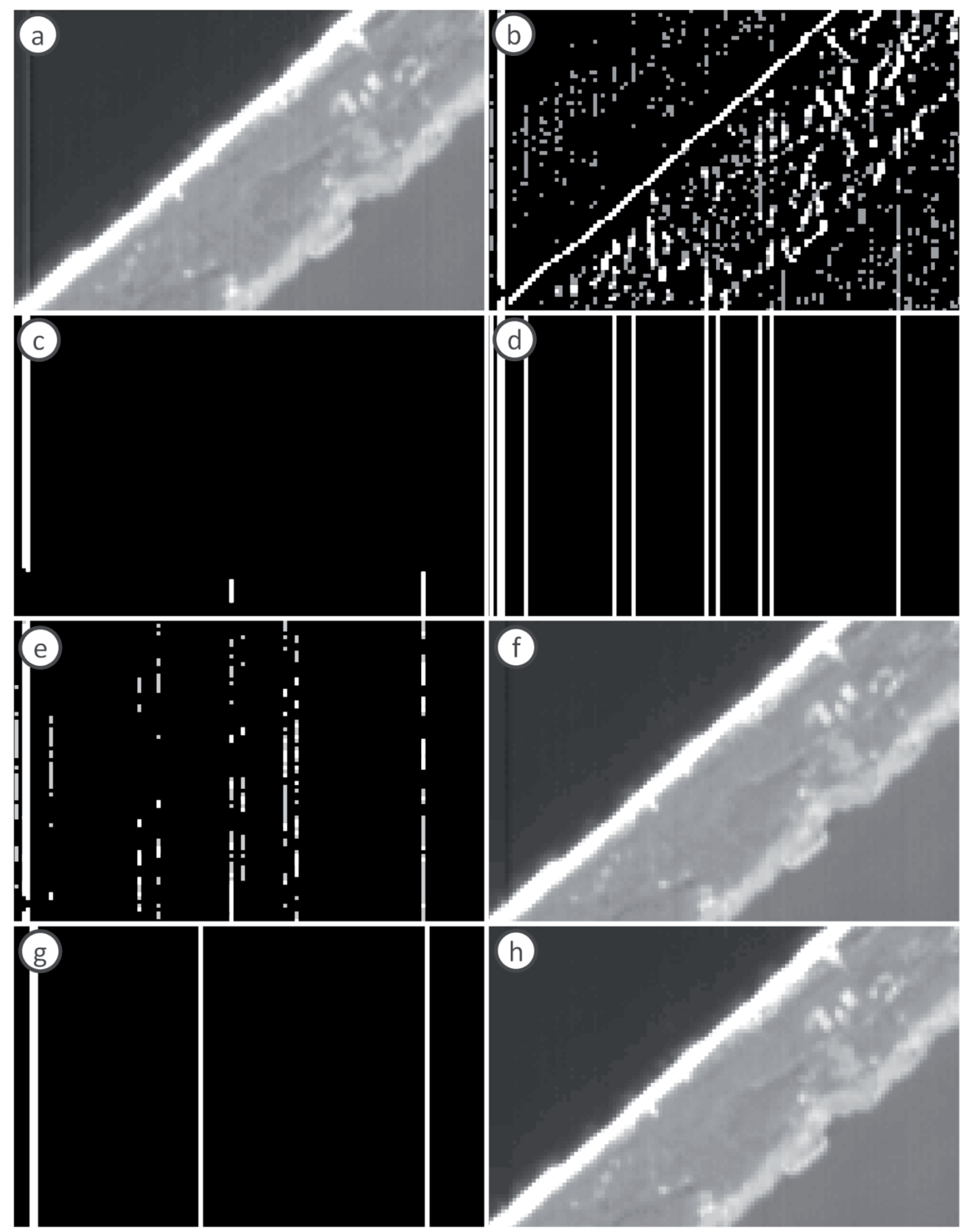

Figure 9. Consecutive steps of vertical lines suppression algorithm a) original image, b) top hat transformation (step 1), c) erosion (step 2), d) dilation (step 3), e) product of binarization and top-hat transformation (step 5), f) image with suppressed bright noise stripes (step 6), g) dark noise stripes (step 9), h) final result (step 12) 
6. Subtracting the result from the original image.

7. Bottom hat transformation using a short horizontal SE $(3 p \times 1 p)$.

8. Erosion using a vertical SE (1p x 13p).

9. Dilation using a long vertical SE (1p x 1495p - depending on the number of lines in the image.

10. Binarization with a threshold of 1 .

11. Product of the result of binarization and bottom-hat transformation (step 7).

12. Adding the result to the original image.

Figure 10 presents the result of the algorithm in a different image. It shows a high efficiency and most importantly, does not affect the shapes of objects in the image.

\subsection{CHRIS Morphological Adaptive Filter}

All three algorithms presented above form a filter called CHRIS Morphological Adaptive Filter (CMAF), dedicated to all noise types in CHRIS satellite images. Figure 11 presents the result of this filtration, compared with the original, 'noised' image.

We can see, that horizontal bad lines are completely eliminated and the number of vertical stripes is significantly decreased. The poorest result is obtained in Figure $11 \mathrm{~b}$ - the image presented in Figure 11a is very 'noised', and vertical stripes sometimes form larger groups, so a small SE used for top hat and bottom hat transformations may not detect them. Nevertheless, the improvement of the image quality is significant.

Figures 12 and 13 below present CMAF results with original images and also, with the Settle method and median filter results.

Figure 12 shows that the Settle method gives the best results (compared to other filters presented, also when compared to CMAF), when it is not affected by the error caused by the occurrence of large, very bright or dark objects in the image.
Figure 13 presents a different case; the image obtained using the Settle method is strongly affected by relatively big clouds in the image. This results in visible bright and dark and relatively broad bands. On the other hand, CMAF produces an image without this kind of error.

The median filter, in both cases, shows relatively poor efficiency - not all noise is eliminated, and it also strongly affects the objects in the image, modifying an important part of the image.

It is important to note that all the processes adopting CMAF algorithms were calculated using BlueNote software - open source freeware created in the Laboratory of Remote Sensing and GIS in Warsaw University of Technology. The software can be downloaded from the laboratory website: http://telesip.gik.pw.edu.pl and used for any purposes. Please, do not hesitate to contact the author of this article about BlueNote.

\section{Conclusions}

CMAF seems to be an interesting solution for CHRIS satellite images filtration. It completely removes horizontal bad lines and, at a minimum, strongly limits the number of vertical stripes. In many cases, the popular Settle method is a very good proposition, as a CHRIS image filtering method, but in cases when the Settle method produces erroneous vertical bands due to the occurrence of large bright or dark objects in the image, CMAF is a very interesting alternative solution. It also removes bright bad lines, which are not removed using Settle method.
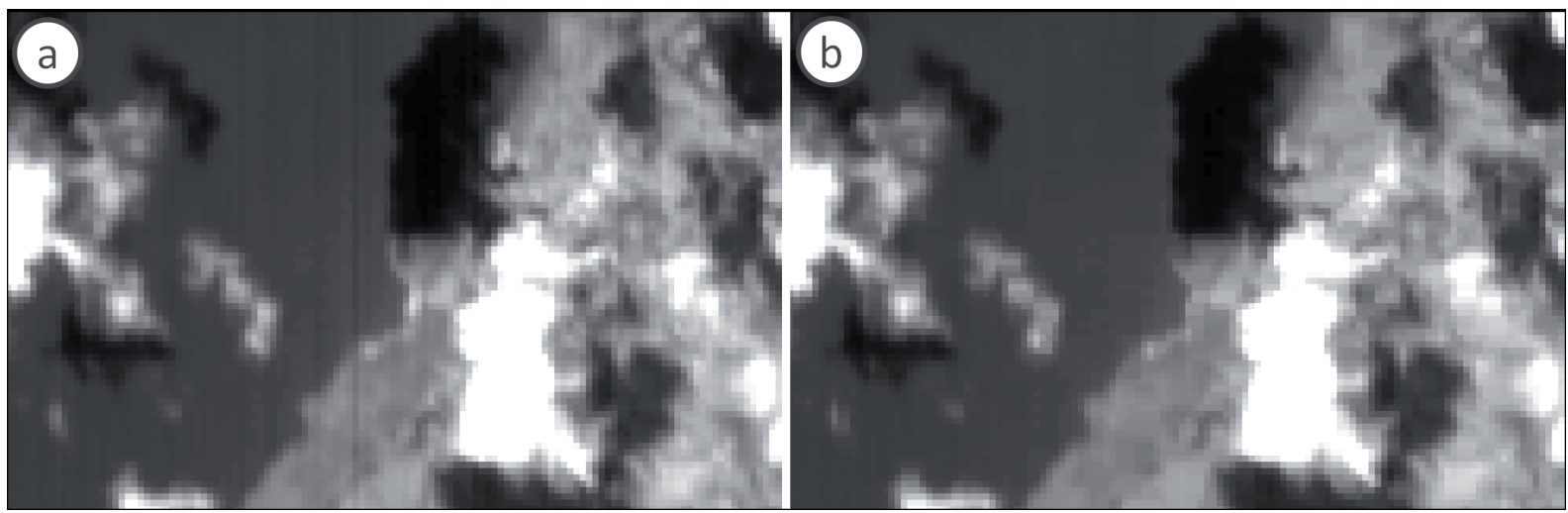

Figure 10. Result of the vertical stripes suppression algorithm, a) original image, b) image after vertical stripes suppression 


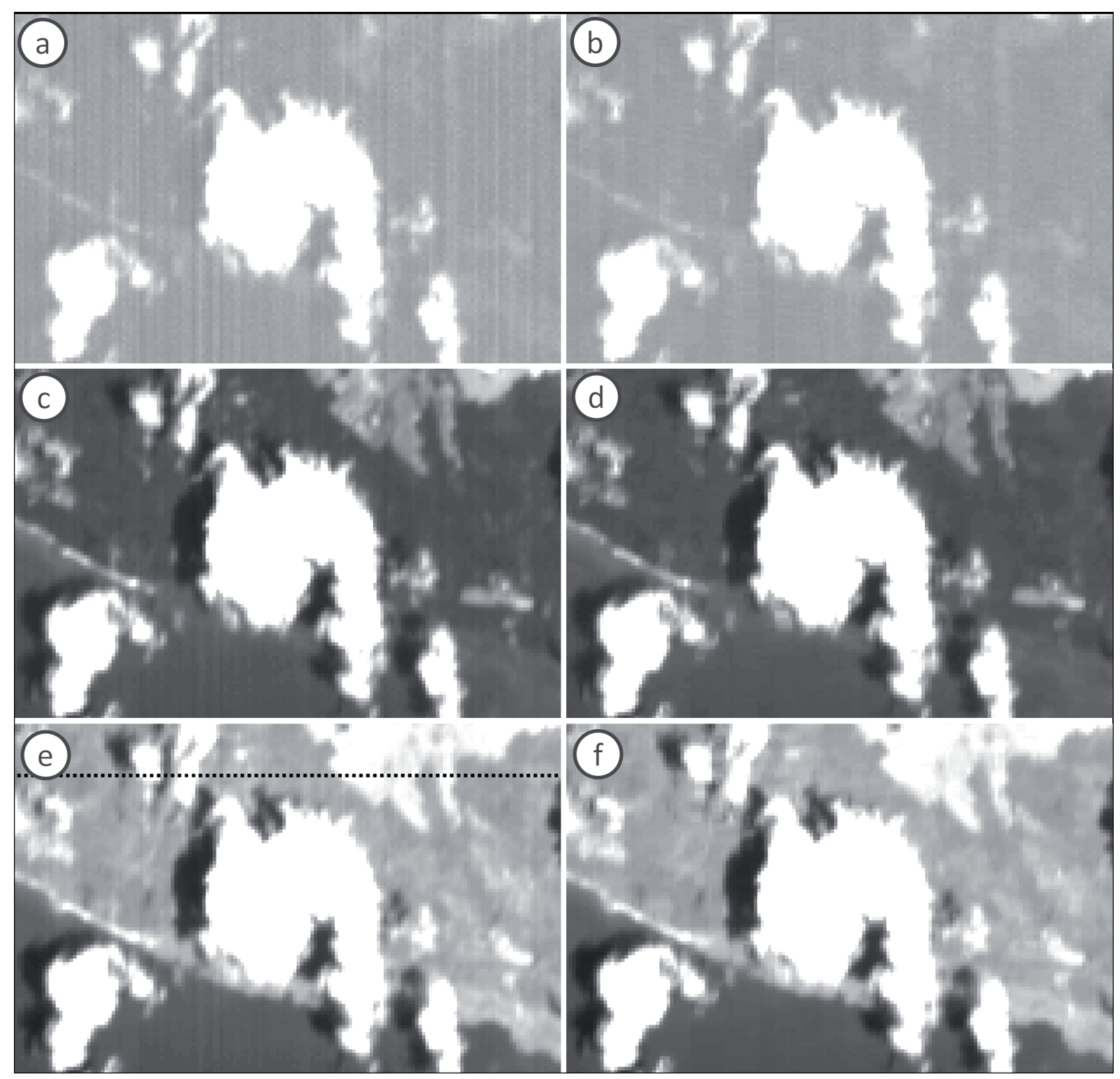

Figure 11. Different CHRIS bands: 11a), 11c) and 11e) original, 11b), 11d) and 11f) after CMAF filtration 


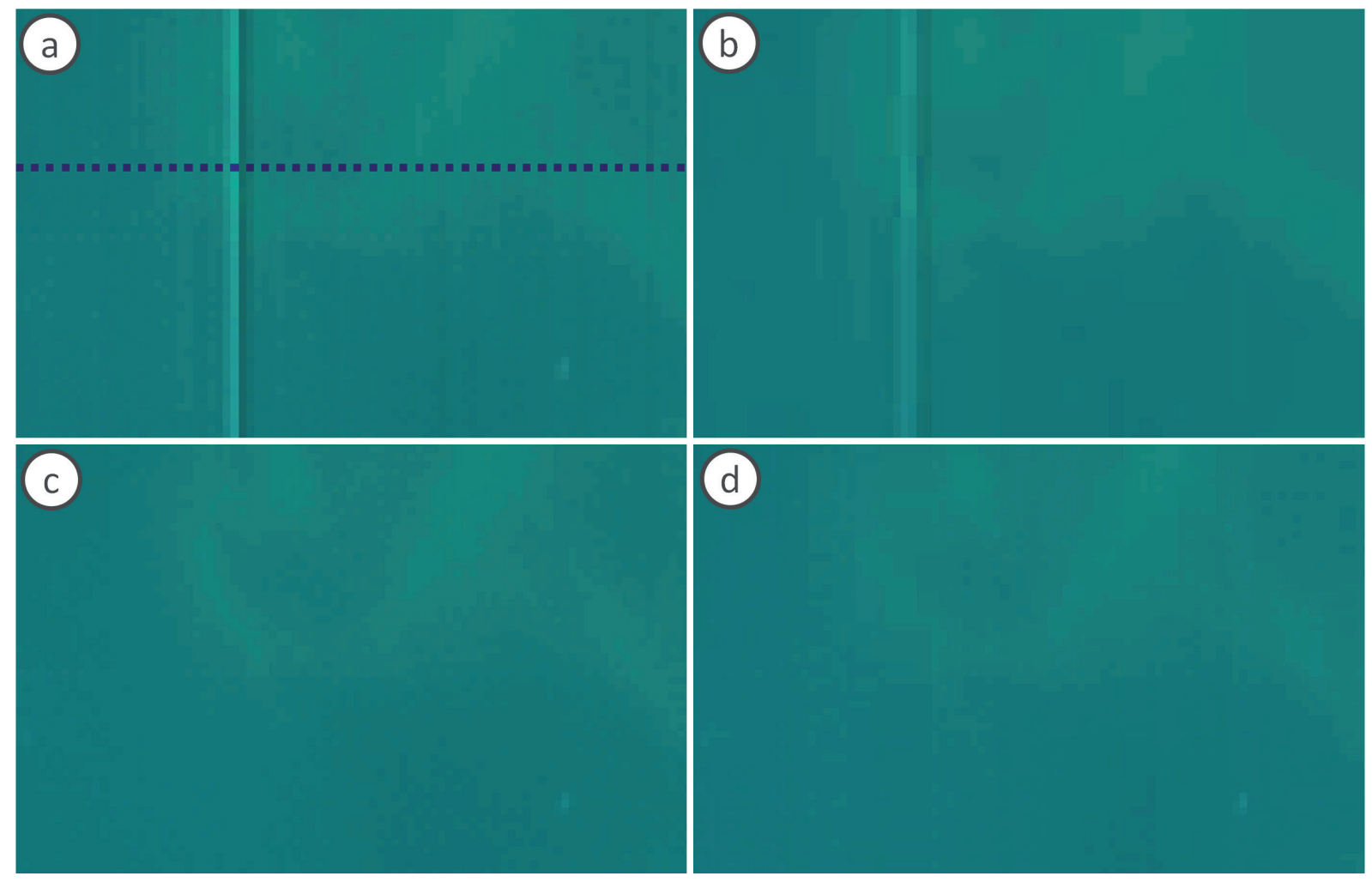

Figure 12. Results of different filtering methods, a) original image, b) median filtration, c) Settle method, d) CMAF

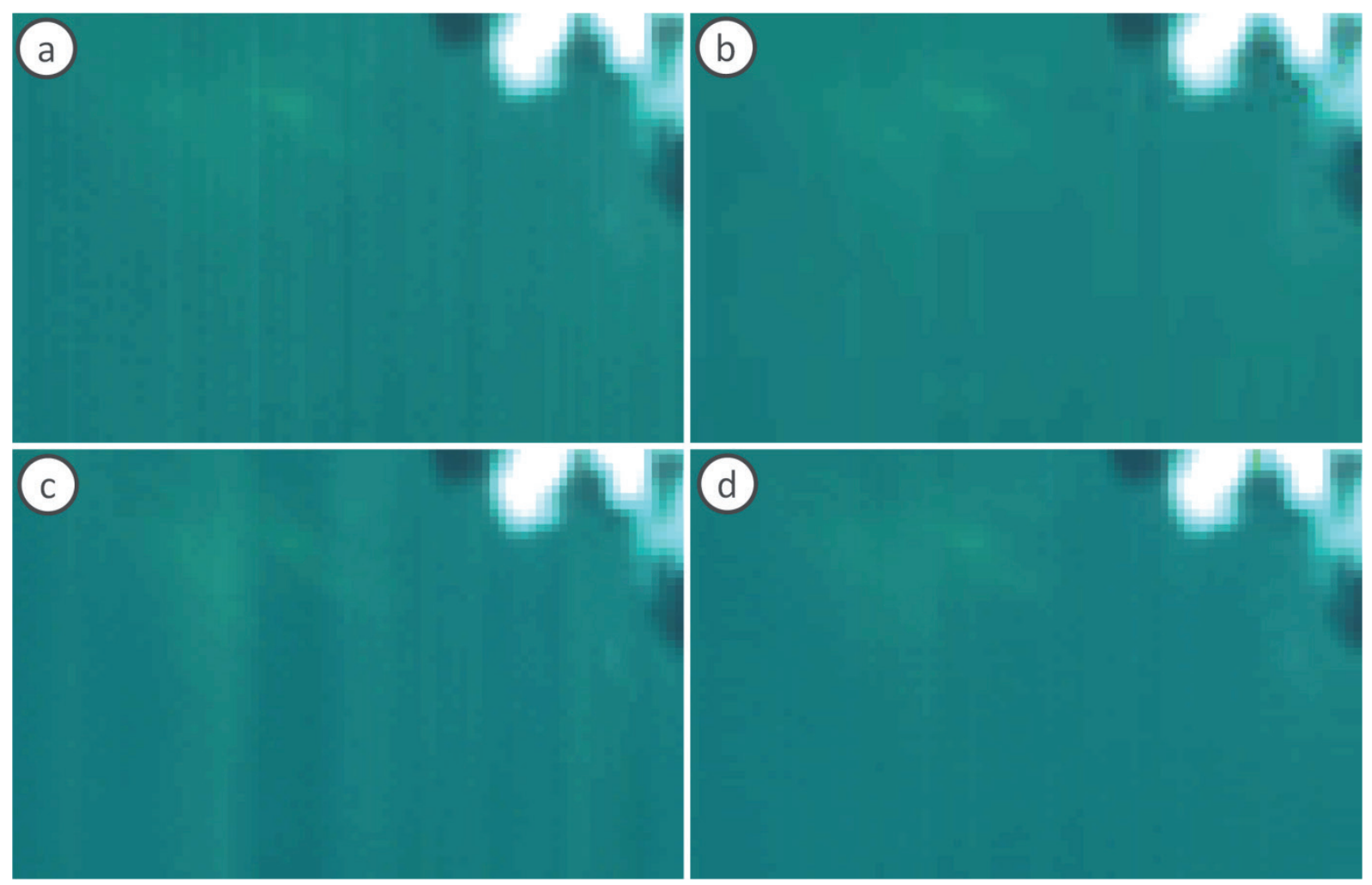

Figure 13. Results of different filtering methods, a) original image, b) median filtration, c) Settle method, d) CMAF 


\section{Reference}

Barducci A., Guzzi D., Marcoionni P. \& Pippi I., 2005, CHRIS-PROBA Performance Evaluation: Signal-ToNoise Ratio, Instrument Efficiency and Data Quality From Acquisitions Over San Rossore (Italy) Test Site. Proc. of the $3^{\text {rd }}$ ESA CHRIS/Proba Workshop, 21-23 March, ESRIN, Frascati, Italy.

Cheng F. \& Venetsanopoulos A. N., 1992, An Adaptive Morphological Filter for Image Processing, IEEE Transactions on Image Processing 1 (4): 533-539.

Cutter M. A., 2004, HDFClean V2 Help, source: www. esa.int.

Garcia J. C. \& Moreno J., 2004, Removal of noises in CHRIS/PROBA images: application to the SPARC Campaign data, Proc of 2nd CHRIS/Proba Workshop, ESA/ESRIN, Frascati, Italy, 28-30 April. ESA SP-578, 2004.

Gomez-Chova L., Alonso L., Guanter L., Calpe J. \& Moreno J., 2008, CHRIS/PROBA Noise Reduction Module, Algorithm Theoretical Basis Document, Development of CHRIS/PROBA modules for the BEAM toolbox, ESA ESRIN Contract No. 20442/07/I-LG.

Haralick R. M., Sternberg S. R. \& Zhuang X., 1987, Image Analysis using Mathematical Morphology, IEEE Transactions on Pattern Analysis and Machine Intelligence 9 (4): 532-550.

Kupidura P., 2006, Zastosowanie wybranych operacji morfologii matematycznej do wydzielania klas pokrycia terenu na zdjęciach satelitarnych [Application of particular mathematical morphology operations for land cover classification of satellite images], phd thesis, Warsaw University of Technology, Warsaw.

Kupidura P., Koza P. \& Marciniak J., 2010, Morfologia matematyczna w teledetekcji [Mathematical morphology in remote sensing], Wydawnictwa Naukowe PWN, Warsaw.

Matheron G., 1967, Eléments pour une théorie des milieux poreux. Masson, Paris.

Matheron G., 1978, Randoms sets and integral equation, Wiley, New York.
Nieniewski M., 1998, Morfologia matematyczna w przetwarzaniu obrazów [Mathematical morphology in image processing], Akademicka Oficyna Wydawnicza PLJ, Warsaw.

Nieniewski M., 2005, Segmentacja obrazów cyfrowych [Digital images segmentation] Metody segmentacji wododziałowej, Akademicka Oficyna Wydawnicza EXIT, Warsaw.

Osińska-Skotak K., Kruk M., Mróz M. \& Ciołkowska M., 2004, Superspektralne dane satelitarne CHRIS/PROBA w ocenie jakości wód jeziornych [Superspectral CHRIS/PROBA satellite date for lake water quality asessment] Archiwum Fotogrametrii, Kartografii i Teledetekcji 14: 425-436.

Serra J., 1982, Image Analysis and Mathematical Morphology, Vol. 1, Academic Press, London.

Serra J., 1986, Introduction to mathematical morphology, Computer Vision Graphics and Image Processing 35 (3): 283-305.

Serra J., 1988, Image Analysis and Mathematical Morphology, Vol. 2: Theoretical Advances, Academic Press, London.

Song J. \& Delp E. J., 1990, The Analysis of Morphological filters with multiple structuring elements, Computer Vision Graphics and Image Processing 50: 308-328.

Sternberg S. R., 1986, Grayscale Morphology, Computer Vision Graphics and Image Processing 35 (3) 333-355.

Stevenson R. L. \& Arce G. R., 1987, Morphological filters: Statistics and further syntactic properties, IEEE Transactions on Circuits and Systems 34 (11): 1292-1305.

Vincent L., 1992, Morphological Area Opening and Closings for Greyscale Images, Proceedings of Shape in Picture'92 - NATO Workshop, Driebergen, The Netherlands, 92, Springer Verlag.

Vincent L., 1993, Grayscale Area Opening and Closing, their efficient implementation and applications, Proceedings of Eurasip Workshop on Mathematical Morphology and its Applications to Signal Processing, Barcelona, Spain. 
